\title{
The Upper Atmosphere and Plasma Imager/the Telescope of Visible Light (UPI/TVIS) onboard the Kaguya spacecraft
}

\author{
M. Taguchi ${ }^{1}$, T. Sakanoi ${ }^{2}$, S. Okano ${ }^{2}$, M. Kagitani ${ }^{2}$, M. Kikuchi $^{3}$, M. Ejiri ${ }^{3}$, I. Yoshikawa ${ }^{4}$, A. Yamazaki ${ }^{5}$, \\ G. Murakami ${ }^{4}$, K. Yoshioka ${ }^{4}$, S. Kameda ${ }^{5}$, W. Miyake ${ }^{6}$, M. Nakamura ${ }^{5}$, and K. Shiokawa ${ }^{7}$ \\ ${ }^{1}$ Rikkyo University, 3-34-1 Nishi-Ikebukuro, Toshima, Tokyo 171-8501, Japan \\ ${ }_{2}^{2}$ Planetary Plasma and Atmospheric Research Center, Tohoku University, Aramaki-aza-aoba, Aoba, Sendai 980-8578, Japan \\ ${ }^{3}$ National Institute of Polar Research, 1-9-10 Kaga, Itabashi, Tokyo 173-8515, Japan \\ ${ }^{4}$ Department of Earth and Planetary Science, The University of Tokyo, 7-3-1 Hongo, Bunkyo, Tokyo 113-0033, Japan \\ ${ }^{5}$ Institute of Space and Astronautical Science, 3-1-1 Yoshinodai, Sagamihara, Kanagawa 229-8510, Japan \\ ${ }^{6}$ School of Engineering, Tokai University, 1117 Kitakaname, Hiratsuka, Kanagawa 259-1292, Japan \\ ${ }^{7}$ Solar-Terrestrial Environment Laboratory, Nagoya University, 3-13 Honohara, Toyokawa, Aichi 442-8507, Japan
}

(Received August 9, 2009; Revised September 11, 2009; Accepted September 11, 2009; Online published January 18, 2010)

\begin{abstract}
The Upper Atmosphere and Plasma Imager (UPI) was placed in a lunar orbit in order to study both the Moon and Earth. The UPI consists of two telescopes: a Telescope of Extreme Ultraviolet (TEX) and a Telescope of Visible Light (TVIS), which are both mounted on a two-axis gimbals system. The TVIS is equipped with fast catadioptric optics and a high-sensitivity CCD to image swift aurora and dark airglow in the terrestrial upper atmosphere. TVIS has a field-of-view equivalent to the Earth's disk as seen from the Moon. The spatial resolution is about $30 \mathrm{~km} \times 70 \mathrm{~km}$ on the Earth's surface at auroral latitudes. The observation wavelengths can be changed by selecting different bandpass filters. Using the images of the northern and southern auroral ovals taken by TVIS, the intensities and shapes of the conjugate auroras will be quantitatively compared. Using the airglow imaging, medium- and large-scale ionospheric disturbances will be studied. In this paper, the instrumental design and performance of TVIS are presented.
\end{abstract}

Key words: Aurora, airglow, imaging, remote sensing, ionosphere, KAGUYA.

\section{Introduction}

Satellite observations of aurora have revealed the entire structure of the auroral oval and the time sequence of an auroral substorm. Most of the aurora observation satellites for in situ measurements of the fields and particles, as well as remote observations of the auroral images, are located in a polar orbit with a perigee of several hundred kilometers and an apogee of 10,000 kilometers or higher. However, in situ measurements cannot distinguish the temporal variation from the spatial variation due to the spacecraft's orbital motion. For imaging observations from an elliptic orbit with a large eccentricity, the observation geometry changes as the satellite orbits the Earth. This orbital motion prevents the satellite from continuously monitoring the aurora at a fixed position. On the other hand, from a lunar orbit at a distance of 60 times the Earth's radius, an almost complete hemisphere of the Earth can be seen. It should be noted that the exact portion of the hemisphere that is visible varies based on the day-night ratio of the hemisphere facing the Moon and the lunar phase.

Imaging observations of aurora have been performed by many satellites, such as ISIS-2 (Anger et al., 1973), KYOKKO (Kaneda, 1979), DE-1 (Frank et al., 1981), VIKING (Anger et al., 1987), AKEBONO (Oguti et al.,

Copyright (c) The Society of Geomagnetism and Earth, Planetary and Space Sciences (SGEPSS); The Seismological Society of Japan; The Volcanological Society of Japan; The Geodetic Society of Japan; The Japanese Society for Planetary Sciences; TERRAPUB
1990), POLAR (Torr et al., 1995), and IMAGE (Burch, 2000). Most of the imagers work in the vacuum ultraviolet region, since, in this wavelength region, dayside scattering sunlight is relatively weak due to absorption by oxygen molecules in the lower thermosphere. A few imagers observed visible light. Higher spatial resolution can be attained using the visible region than using the vacuum ultraviolet region.

Conjugacy and non-conjugacy of auroras in the northern and southern polar regions have provided information on the structure of the magnetosphere and the acceleration mechanism of the auroral particles. Ground-based observations of conjugate auroras have improved our understanding of these topics (cf. DeWitt, 1962; Stenbaek-Nielsen et al., 1972; Sato and Saemundsson, 1987). Belon et al. (1969) reported the results of a unique conjugate auroral observation made from airplanes. To date, this has been the only attempt to use airborne instruments. Pairs of conjugate points that are suitable for ground-based observations are very limited due to asymmetry in the land distribution between the northern and southern polar regions. Furthermore, during disturbed magnetic conditions, the conjugate points move a long distance away from the positions under quiet conditions. These conditions make ground-based observation of conjugate aurora very difficult. Østgaard et al. (2004) compared the auroral images simultaneously taken by the IMAGE and POLAR satellites in both hemispheres. Two identical imagers onboard the two satellites in different or- 
bits are necessary for precise comparison of auroral features and intensities.

Imaging observations of airglow by monochromatic allsky imagers with high-sensitivity CCD cameras have been an important technique in the studies of the global dynamics of the upper atmosphere (cf. Taylor and Hapgood, 1990; Shiokawa et al., 1999). These imagers revealed various scales of wavy structures as small perturbations in the airglow images. Mendillo et al. (1997) investigated traveling ionospheric disturbances (TIDs) as shown in all-sky images of 630-nm OI airglow. Kubota et al. (2000) visualized the TIDs propagating over Japan by a network of monochromatic all-sky imagers. Even though the four imagers are distributed over Japan from south to north, only a part of the TIDs can be shown. The origin, termination, and spatial extent of TIDS are still unknown, because they are out of the field-of-view of the imager network.

A far ultraviolet image of the subtropical airglow corresponding to the equatorial anomaly (EA) was first taken from Apollo 16 (Carruthers and Page, 1976). Airglow imagery from both space and ground is one of the useful methods in the study of plasma instabilities in EA. Sagawa et al. (2003) discuss the longitudinal wavy structures seen in the low latitude OI 135.6-nm airglow emissions obtained by the IMAGE/FUV instrument. The conjugacy of the electron density depletion in the low latitude regions in both hemispheres is also shown by simultaneous ground-based observations of the OI 630.0-nm airglow emission at Sata, Japan and Darwin, Australia by Otsuka et al. (2004). Only a part of the ionospheric disturbances is seen by a groundbased imager. It is expected that further study on plasma instabilities in the low latitudes will be promoted by satellite imagery of airglow.

KAGUYA is the Japanese lunar orbiter launched on September 14, 2007 with a polar orbit and an altitude of $100 \mathrm{~km}$. A major purpose of this mission was to obtain comprehensive data that can be used to study the origin and evolution of the Moon and moon utilization in the future (Sasaki et al., 2003; Kato et al., 2008). KAGUYA observes the surface composition, topography, underground structure, magnetic field anomaly, and gravity all over the Moon using remote sensing. Also, in situ measurement of plasma density, electric and magnetic fields, and high-energy particles are performed. Two subsatellites, OKINA and OUNA, which orbit in elliptic orbits, provide support for communication between KAGUYA and the ground and gravity measurements. The nominal mission life is 1 year.

The Upper Atmosphere and Plasma Imager (UPI) onboard KAGUYA is composed of two imagers and a gimbals mount that points the imagers in the desired direction. Unlike the other instruments onboard KAGUYA, UPI looks at the Earth from the lunar orbit in order to visualize the Earth's upper atmosphere and plasmasphere. The EUV telescope (UPI-TEX) takes images of the plasmasphere and polar wind using $\mathrm{HeI}$ and OII emissions in the EUV region (Yoshikawa et al., 2008), and the visible telescope (UPITVIS) takes global-scale images of aurora and airglow from the lunar orbit to study the subjects discussed above. This paper presents the instrumental design and performance of TVIS.

\section{Instrument Design}

TVIS optics are designed to have high sensitivity in order to visualize monochromatic signatures of fast moving aurora and the faint airglow. An aurora with a typical intensity of $10 \mathrm{kR}$ must be imaged with an exposure time of $10 \mathrm{~s}$, and the darker airglow with an intensity of $100 \mathrm{R}$ to $1 \mathrm{kR}$ requires an exposure time of 100 to $1000 \mathrm{~s}$. The field-ofview of $2.38^{\circ} \times 2.38^{\circ}$ is designed to fit the Earth's viewing angle of $2^{\circ}$ from the lunar orbit, and the spatial resolution is $0.005^{\circ}$, which corresponds to horizontal distances of $30 \mathrm{~km}$ $\times 70 \mathrm{~km}$ on the Earth's surface at auroral latitudes. This spatial resolution is high enough to resolve medium-scale auroral structures and disturbances seen in airglow.

Catadioptric optics that consists of six lenses with two reflecting surfaces were adopted to meet both the highsensitivity and spatial resolution requirements for the wide spectral coverage. The optical design is shown in Fig. 1, and a picture of the TVIS is shown in Fig. 2. The clear aperture size of the optics is $136 \mathrm{~mm}$ with an F-number of 2.35. There is the secondary mirror at the center of the first lens, and, therefore, the effective light collecting power is 0.75 of that of the full clear aperture. The lenses are made of quartz, F2 and BK7, and they are all antireflection (AR) coated. Figure 3 shows spot diagrams and modulation transfer functions (MTFs) of the TVIS optics at a wavelength of $557.7 \mathrm{~nm}$.

Interference filters are used to select an emission line of the aurora and the airglow. The target emissions, wavelengths, bandwidths, and filter transmittance are listed in Table 1. The target auroral and airglow emissions are $\mathrm{N}_{2}^{+}$ 1NG $427.8 \mathrm{~nm}$, OI $557.7 \mathrm{~nm}, \mathrm{NaD} 589.3 \mathrm{~nm}$, OI $630.0 \mathrm{~nm}$, and $\mathrm{OH}$ and $\mathrm{N}_{2}$ band emissions. Only the filter at the fifth position is a longpass filter, while the other filters are all narrow bandpass filters. The interference filters and a shutter plate are mounted in a filter wheel, which is driven by a stepping motor. The position of filters is detected by a potentiometer that rotates the same angle as the wheel rotates. The structure of the telescope is made of aluminum alloy, titanium alloy, Super Inver, polyimide-based polymer (VESPEL), and carbon fiber reinforced plastic (CFRP). The most appropriate material for each part is selected based on the required thermal and mechanical properties. The weight of the TVIS optics is $3.05 \mathrm{~kg}$.

The image sensor is a back-illuminated, frame transfer CCD cooled by a Peltier cooler. The surfaces of the window and the CCD are AR-coated to maximize quantum efficiency of $\mathrm{CCD}$ in the visible and near infrared regions. In order to reduce thermal noise when the CCD is operational, it is cooled down and stabilized at about $-40^{\circ} \mathrm{C}$ within a few minutes after the cooler is turned on. A circular plate with a diameter of $200 \mathrm{~mm}$ and an a chromate conversion coating (Alocrom 1000) is equipped with the hightemperature side of the Peltier cooler for radiative cooling. The CCD has $1024 \times 1024,13-\mu \mathrm{m}$ square pixels, which are converted to $512 \times 512,26-\mu \mathrm{m}$ square super pixels using 2 $\times 2$ pixel binning. The data depth is 12 bits, while the data size and depth yield of the raw image data size is $390 \mathrm{kB}$.

The TVIS and TEX telescopes are mounted on the twoaxis gimbals mount that tracks the Earth as shown in figure 1 of Yoshikawa et al. (2008). The azimuth axis can- 


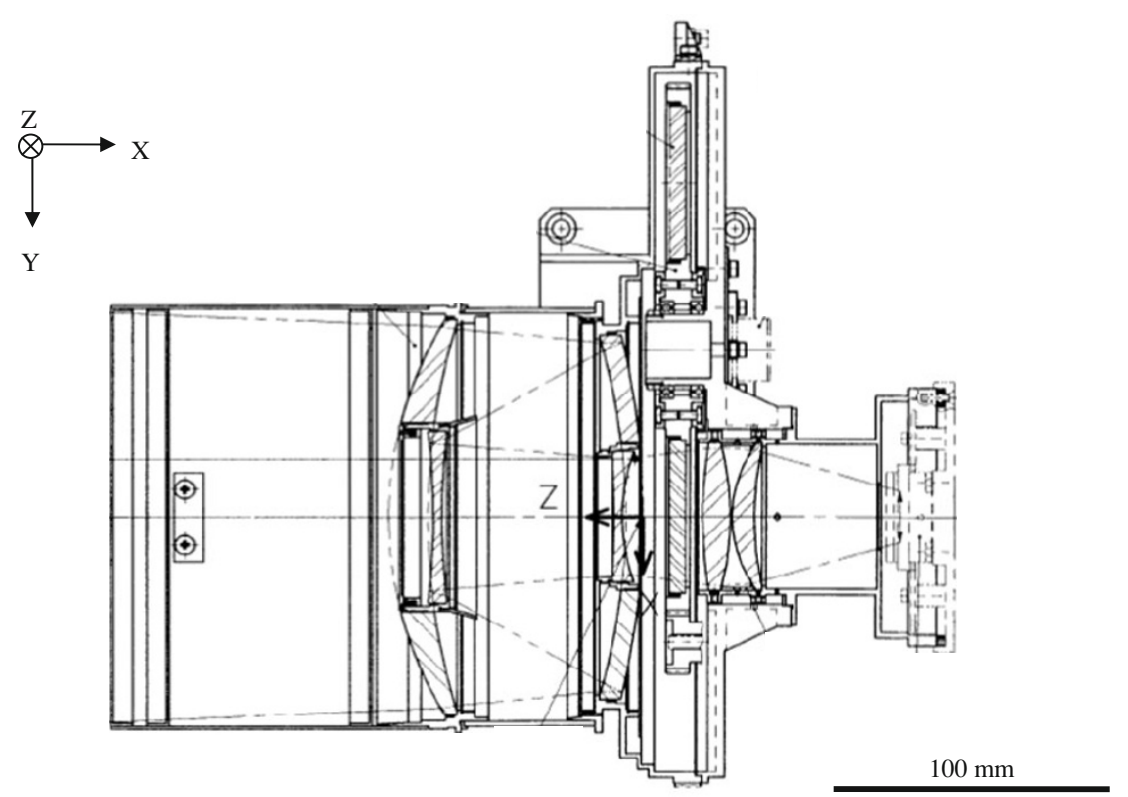

Fig. 1. Optical layout of TVIS.

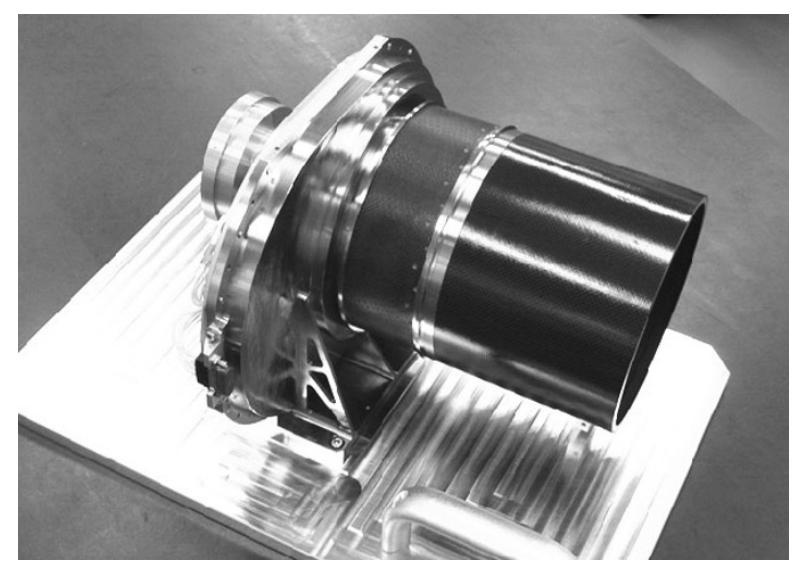

Fig. 2. A picture of the TVIS without the MLI and the radiator plate.

cels the orbital motion of the spacecraft, and the elevation axis cancels the orbital motion of the Moon around the Earth. The rotation speed of the azimuth is approximately $100 \mathrm{~min} /$ revolution, while the elevation axis rotates at approximately 27 days/revolution. Since the TVIS has a finer spatial resolution than the TEX, the spatial resolution of the TVIS restricts the tracking accuracy of the gimbals. The gimbals with the telescopes are mounted on an extendable arm, which is folded and rigidly fixed to the baseplate so that it keeps the telescopes safe from vibration during the launch. After the spacecraft enters the lunar orbit, the arm is extended to deploy the gimbals to the observation position.

TVIS is not operational under sunlit conditions, and therefore a high-performance hood to avoid solar light contamination is unnecessary. However, TVIS has a small hood to eliminate moonlight reflected by the spacecraft body and to prevent the front lens from being overcooled by radia- tive cooling. TVIS has two sets of operational heaters and temperature sensors to keep its temperature within certain limits. The first temperature sensor is located on the telescope tube close to the front lens and requires $7.2 \mathrm{~W}$ of power. The turn-on/off temperatures are $-5^{\circ} \mathrm{C} / 0^{\circ} \mathrm{C}$. The other temperature sensor is located on the rear telescope tube and requires $2.5 \mathrm{~W}$ of power. The turn on/off temperatures are $-45^{\circ} \mathrm{C}-40^{\circ} \mathrm{C}$. All of the parts of the UPI are covered by a multilayer insulator for thermal stability.

A bright object sensor (BOS) is equipped with TVIS to protect against accidental direct exposure of the strong solar radiation to the detectors of TVIS and TEX. BOS is a simple light detector that consists of a pinhole and a photodiode, and is only sensitive to light as bright as the Sun. The field-of-view is about $60^{\circ}$. When BOS detects the Sun, TVIS and TEX are shut down, and their fields-of-view are aimed to a safe direction.

Only a pre-amplifier for the CCD is contained in the case attached on the TVIS telescope. Most of the electronics that drive the CCD and acquire data from the CCD are mounted in the UPI-E. The UPI-E and TVIS are connected by flexible wire harnesses that go through the inside of the axes.

TVIS is operational when the spacecraft is in the nightside of the Moon and more than half of the nightside of the Earth is visible from the Moon, as shown in Fig. 4. This condition occurs for approximately 1 week every month, except when the $\beta$-angle, that is, the angle between the Sun's direction and the plane of the spacecraft's orbit, is approximately $0^{\circ}$ or $90^{\circ}$. When the $\beta$-angle is around $0^{\circ}$, the UPI can see the Earth only from the sunlit position. When the $\beta$-angle is around $90^{\circ}$, the spacecraft is always sunlit. The total period of observation windows of TVIS is about one-tenth of the total mission life.

To reduce the data produced, TVIS has two exclusive data handling functions: differentiation and cut-out. The 
(a)

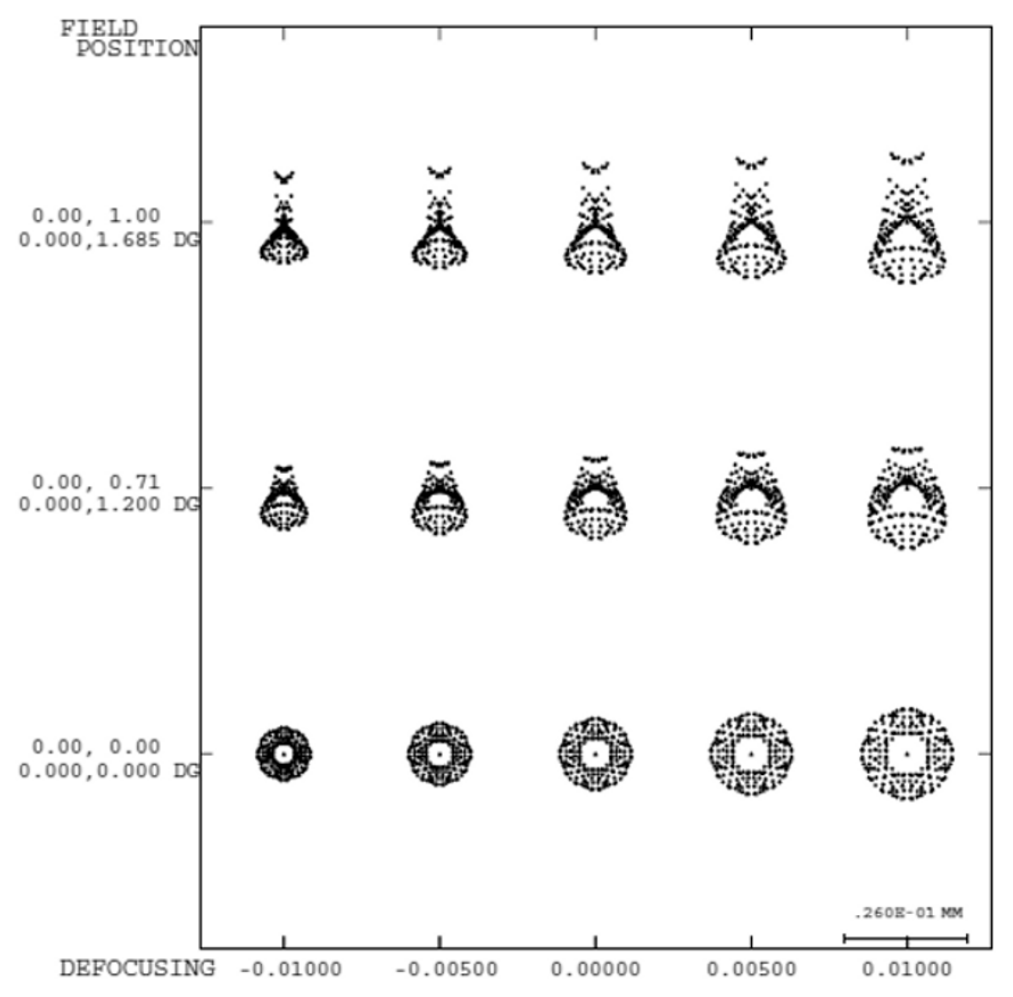

(b)

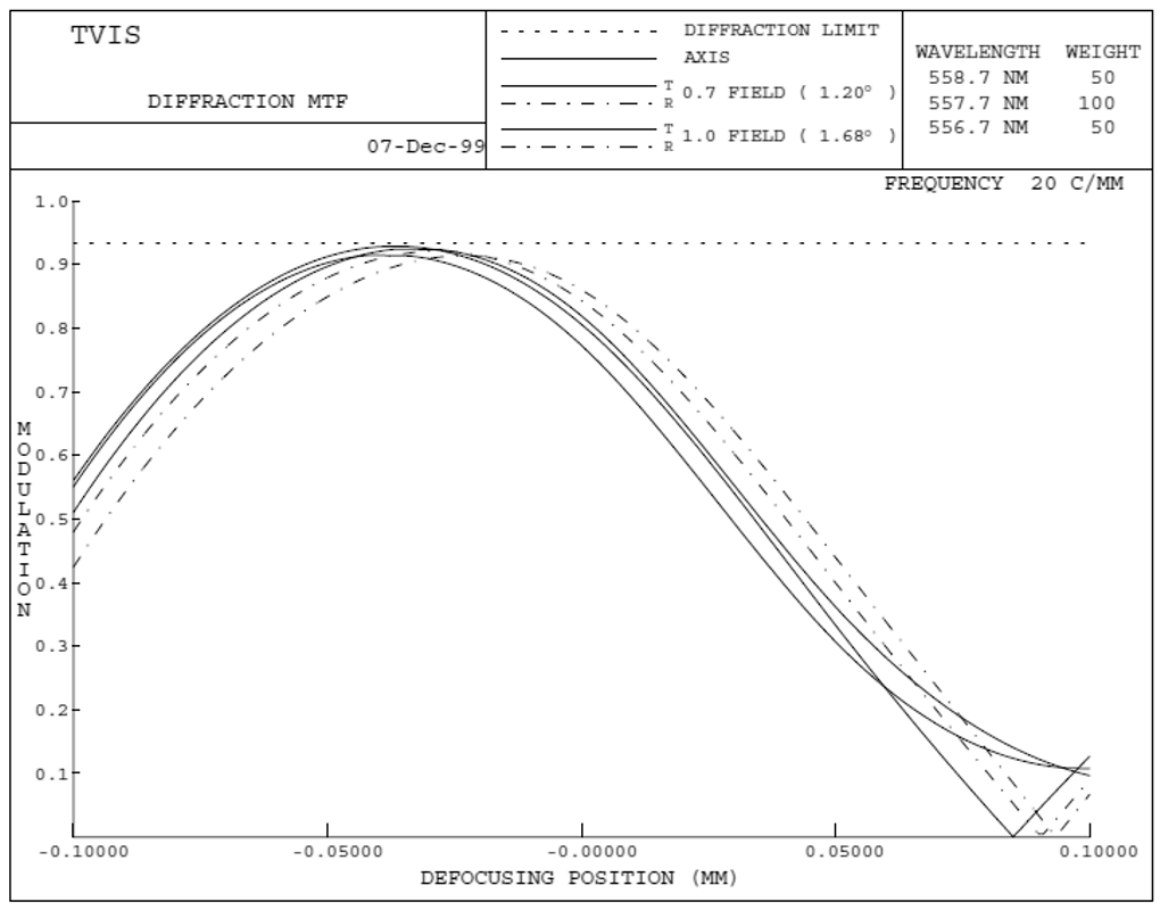

Fig. 3. Spot diagrams (a) and MTFs at a spatial frequency of $20 \mathrm{c} / \mathrm{mm}$ as a function of the defocusing at a wavelength of $557.7 \mathrm{~nm}$ (b).

differentiation function takes the difference between two successive images using 4 bits. By transforming the first full image into 12 bits and the successive difference image into 4 bits, the data amount is reduced to about one-third of the original amount. This method is effective for images that change little within the data-sampling period. The cutout function removes the area of interest, when the nightside of the Earth is imaged, and only transfer that area's data. The area to be cut-out can be freely selected by a command.
This function reduces the data amount to less than one-half of the original amount.

\section{Test Results}

\subsection{EMC test}

UPI uses four stepping motors: two are fixed on the extendable arm of the gimbals, one on the azimuth axis of the gimbals, and the final one on the TVIS. Although they are magnetically shielded by permalloy plates, significant 
Table 1. Targets and filter specifications.

\begin{tabular}{clccc}
\hline Filter position & Target & Wavelength [nm] & Filter bandwidth (FWHM) [nm] & Peak transmittance \\
\hline 1 & $\mathrm{~N}_{2}^{+} 1 \mathrm{NG}$ & 427.8 & 2.8 & 0.45 \\
2 & $\mathrm{OI}$ & 557.7 & 1.6 & 0.55 \\
3 & $\mathrm{NaD}$ & 589.3 & 3.5 & 0.60 \\
4 & $\mathrm{OI}$ & 630.0 & 2.0 & 0.59 \\
5 & $\mathrm{OH}, \mathrm{N}_{2}$ & $730-800$ & - & 0.98 \\
6 & Shutter & - & - & - \\
\hline
\end{tabular}

Table 2. Vibration and shock test levels.

\begin{tabular}{|c|c|c|c|c|}
\hline Test item & Axis & Frequency $[\mathrm{Hz}]$ & Acceleration [G] & \\
\hline \multirow{3}{*}{ Sine-wave vibration } & Y 7 & $5-50$ & 12.5 & \multirow{3}{*}{ Scan speed 2 [oct/min] } \\
\hline &,$Z$ & $5-100$ & 7.5 & \\
\hline & $\mathrm{X}$ & $5-100$ & 12.5 & \\
\hline \multirow{8}{*}{ Random vibration } & \multirow{4}{*}{$\mathrm{Y}, \mathrm{Z}$} & $20-100$ & 7.66 [dB/oct] & \multirow{8}{*}{ Duration 80 [sec] } \\
\hline & & $100-800$ & $0.06\left[\mathrm{G}^{2} / \mathrm{Hz}\right]$ & \\
\hline & & $800-2000$ & $-5.89[\mathrm{~dB} / \mathrm{oct}]$ & \\
\hline & & Overall & $8.54\left[\mathrm{G}_{\mathrm{rms}}\right]$ & \\
\hline & \multirow{4}{*}{$\mathrm{X}$} & $20-54$ & 9 [dB/oct $]$ & \\
\hline & & $54-700$ & $0.1\left[\mathrm{G}^{2} / \mathrm{Hz}\right]$ & \\
\hline & & $700-2000$ & $-6.6[\mathrm{~dB} / \mathrm{oct}]$ & \\
\hline & & Overall & $10.38\left[\mathrm{G}_{\mathrm{rms}}\right]$ & \\
\hline \multirow{2}{*}{ Shock } & \multirow{2}{*}{$\mathrm{X}, \mathrm{Y}, \mathrm{Z}$} & $100-800$ & 13.7 [dB/oct] & \multirow{2}{*}{ Repetition positive 1 negative 1} \\
\hline & & $800-4000$ & $800\left[\mathrm{G}_{\mathrm{srs}}\right]$ & \\
\hline
\end{tabular}

Definition of axes is shown in Fig. 1.

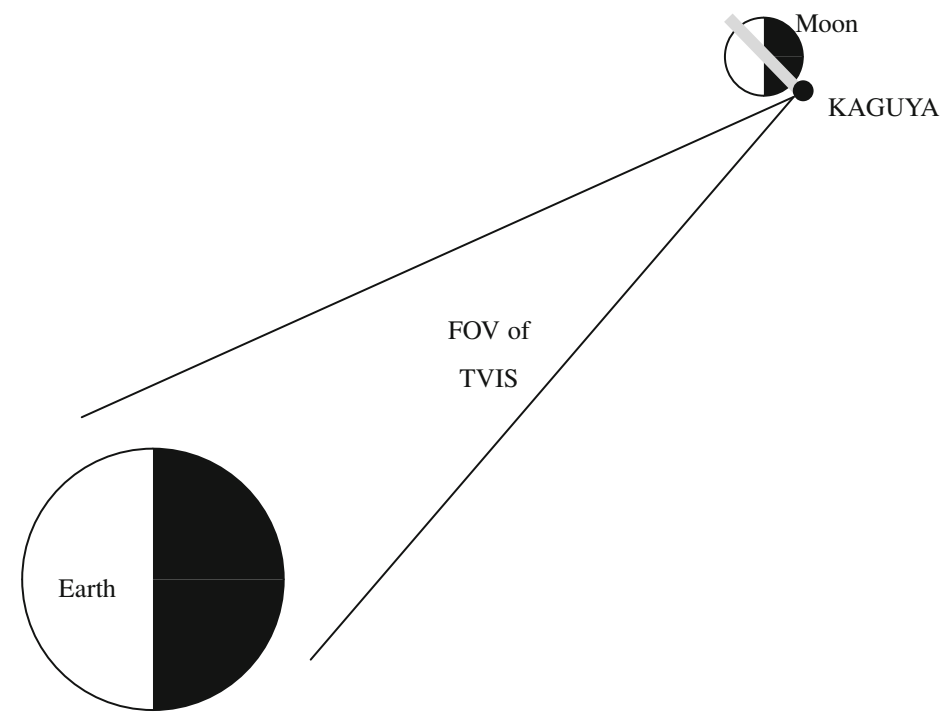

Fig. 4. Observation geometry of UPI-TVIS.

magnetic fields remain from the magnets used in the motors. The residual magnetic fields satisfy the EMC standard for the static magnetic field, but do not satisfy that of the variable magnetic field. However, magnetic interference to the other magnetically sensitive instruments can be avoided by observation time sharing. The other EMC items, such as conductive and radiative electric noises, were tested and cleared.

\subsection{Vibration and shock tests}

The CCD and optics used in TVIS were tested separately by the manufacturer under a mechanical environment estimated by a mathematical model and the mechanical inter- face condition. Vibration and shock tests were performed again for the fully integrated UPI. The test conditions are listed in Table 2. UPI cleared all of the vibration and shock tests. It was also confirmed that the UPI can withstand the mechanical shock generated when the extendable arm is unlocked.

\subsection{Thermal vacuum test}

A thermal vacuum test was performed to confirm the function of the UPI in a vacuum environment. For the TVIS, the test mainly focused on the cooling capability and the temperature distribution of the TVIS under extreme thermal conditions with several thermal cycles. Although 
Table 3. Spot sizes at five positions on the focal plane $[\mu \mathrm{m}]$.

\begin{tabular}{cccccc}
\hline Wavelength [nm] & Center & Upper left & Upper right & Lower left & Lower right \\
\hline 427.8 & 10 & 10 & 8 & 11 & 11 \\
557.7 & 8 & 9 & 10 & 6 & 8 \\
589.3 & 7 & 8 & 9 & 6 & 7 \\
630.0 & 7 & 8 & 7 & 6 & 7 \\
$730-800$ & 12 & 18 & 11 & 13 & 10 \\
\hline
\end{tabular}

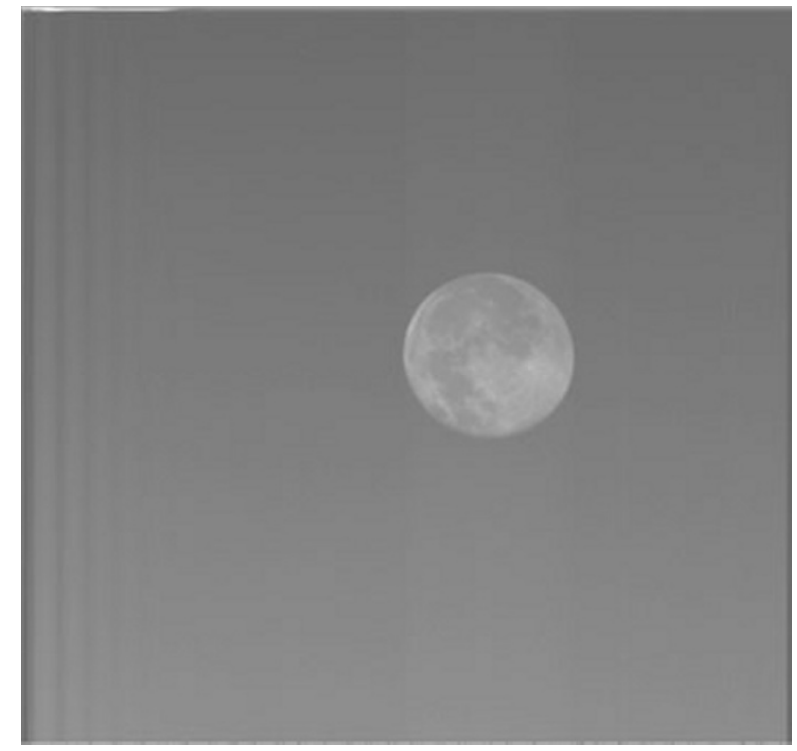

Fig. 5. A test image of the Moon taken by TVIS with the 427.8-nm filter.

simulation of the solar light and deep space is limited in a space chamber, it was confirmed that the Peltier cooler functions as expected both in the coldest and hottest cases, and that the heater system can keep the TVIS temperatures above the lower limit. An EMC test between the TVIS and the TEX was also conducted during the thermal vacuum test. A little noise was seen in TEX data, when the Peltier cooler of the TVIS is turned on and off during the period of TEX data acquisition. This interference is not very severe, because for most of the operational periods, the TVIS and TEX are not turned on at the same time.

\subsection{Image quality}

The imaging performance of the optical system was checked by the manufacturer. Measured spot sizes of a collimator with a focal length of $3.5 \mathrm{~m}$ are listed in Table 3. All the spot sizes are much smaller than the super pixel size of $26 \mu \mathrm{m}$. The focus position was also measured using the collimator, and the CCD was precisely placed at the focal position that takes into consideration the difference between atmospheric and vacuum conditions. Figure 5 is an image of the Moon taken by TVIS to confirm the image quality. Note that the image has large dark noise, because the cooler cannot be turned on due to the existence of water vapor. No pixel defects were found at the time of prelaunch calibration.

\subsection{Sensitivity}

The sensitivity of the TVIS is calibrated by a NIST traceable integrating sphere of the National Institute of Polar Re-
Table 4. Sensitivity of the TVIS.

\begin{tabular}{cc}
\hline Wavelength $[\mathrm{nm}]$ & Sensitivity $[\mathrm{cts} / \mathrm{kR} / \mathrm{s} /$ pixel $]$ \\
\hline 427.8 & 16 \\
557.7 & 23 \\
589.3 & 28 \\
630.0 & 26 \\
$730-800$ & - \\
\hline
\end{tabular}

search. Accuracy in the intensity of the integrating sphere is better than $1 \%$ for the whole wavelength range covered by the TVIS. The results are shown in Table 4 . The sensitivity falls off both in the shorter and longer wavelength limits due to the quantum efficiency of the CCD. The other variation in the sensitivity is due to differences in transmittance of the interference filters. From the calibration results, a signal with 1,600-2,800 counts per pixel is expected for a 10 -s exposure of a 10-kR aurora. Flat-fielding data for each wavelength were obtained in the calibration. On the other hand, the typical dark noise and read-out noise of CCD are $0.24 \mathrm{e}^{-} / \mathrm{pixel} / \mathrm{s}$ at $-30^{\circ} \mathrm{C}$ and $2 \mathrm{e}_{\mathrm{rms}}^{-}$at read-out frequency of $20 \mathrm{kHz}$, respectively.

\section{Operation}

Conjugate aurora observation is conducted in the spring and fall equinox seasons when auroras in both northern and southern hemispheres can be observed, while airglow observation is conducted during the other periods. Auroral images are taken with a high time resolution and short exposure time. Due to the limitations of the data transfer speed, auroral observation cannot continue for successive passes, though UPI-E has a memory of $32 \mathrm{MB}$ where data from the TVIS are stored. For airglow observation, a long exposure time is required, but blooming due to strong light from the dayside of the Earth prevents long exposure. Therefore, the equivalent long exposure time is achieved by stacking many images with short exposure times.

A special operation to detect the lunar sodium atmosphere is planned using the sodium filter of TVIS when the spacecraft is in the nightside of the Moon and the Earth cannot be seen from the Moon. Changes in sodium $589 \mathrm{~nm}$ emission resonantly scattered by the lunar sodium atmosphere and tail may be observed while the spacecraft goes through the Earth's magnetosphere. In this optional observation, the gimbals are operated in either pointing mode to see the antisolar direction or in fixed mode to scan the space.

\section{Summary}

The UPI-TVIS on-board the KAGUYA is designed to take images of the terrestrial aurora and airglow in the visi- 
ble to near-infrared regions from a lunar orbit using fast optics and a high-sensitivity CCD. Observations using the UPI are ongoing, and the TVIS will provide information on the conjugate aurora, auroral substorms and the thermospheric dynamics, including plasma instabilities and medium- and large-scale ionospheric disturbances. Observed image data are being analyzed, and the first results are presented in Kagitani et al. (2009).

Acknowledgments. The authors thank all the members of the SELENE (KAGUYA) mission team for their support in developing UPI.

\section{References}

Anger, C. D., T. Fancott, J. McNally, and H. S. Kerr, ISIS-2 scanning auroral photometer, Appl. Opt., 12, 1753-1766, 1973.

Anger, C. D., S. K. Babey, A. Lyle Broadfoot, R. G. Brown, L. L. Cogger, R. Gattinger, J. W. Haslett, R. A. King, D. J. McEwen, J. S. Murphree, E. H. Richardson, B. R. Sandel, K. Smith, and A. Vallance Jones, An ultraviolet auroral imager for the Viking spacecraft, Geophys. Res. Lett., 14, 387-390, 1987

Belon, A. E., J. E. Maggs, T. N. Davis, K. B. Mather, N. W. Glass, and G. F. Hughes, Conjugacy of visual auroras during magnetically quiet periods, J. Geophys. Res., 74, 1-28, 1969.

Burch, J. L., IMAGE mission overview, Space Sci. Rev., 91, 1-14, 2000.

Carruthers, G. R. and T. Page, Apollo 16 far ultraviolet imagery of the polar auroras, tropical airglow belts, and general airglow, J. Geophys. Res., 81, 483, 1976.

DeWitt, R. N., The occurrence of aurora in geomagnetically conjugate areas, J. Geophys. Res., 68, 1347-1352, 1962.

Frank, L. A., J. D. Craven, K. L. Ackerson, M. R. English, R. H. Eather, and R. L. Carovillano, Global auroral imaging instrumentation for the Dynamics Explorer mission, Space Sci. Instr., 5, 369-393, 1981.

Kagitani, M., M. Taguchi, A. Yamazaki, I. Yoshikawa, G. Murakami, K. Yoshioka, S. Kameda, F. Ezawa, T. Toyota, and S. Okano, First optical observation of the Moon's sodium exosphere from the lunar orbiter SELENE (Kaguya), Earth Planets Space, 61, 1025-1029, 2009.

Kaneda, E., Auroral TV observation by KYOKKO, Proc. Jpn. IMS Symp., 146, 1979.

Kato, M., S. Sasaki, K. Tanaka, Y. Iijima, and Y. Takizawa, The Japanese lunar mission SELENE: Science goals and present status, Adv. Space Res., doi:10.1016/j.asr.2007.03.049, 2008 (in press).

Kubota, M., K. Shiokawa, M. K. Ejiri, Y. Otsuka, T. Ogawa, T. Sakanoi, H. Fukunishi, M. Yamamoto, S. Fukao, and A. Saito, Traveling ionospheric disturbances observed in the OI 630-nm nightglow images over Japan by using a multipoint imager network during the FRONT campaign,
Geophys. Res. Lett., 27, 4037-4040, 2000.

Mendillo, M., J. Baumgardner, D. Nottingham, J. Aarons, B. Reinisch, J. Scali, and M. Kelley, Investigations of thermospheric-ionospheric dynamics with $6300-\AA \AA$ images from the Arecibo Observatory, J. Geophys. Res., 102, 7331-7343, 1997.

Oguti, T., E. Kaneda, M. Ejiri, S. Sasaki, A. Kadokura, T. Yamamoto, K. Hayashi, R. Fujii, and K. Makita, Studies of aurora dynamics by Aurora-TV on the Akebono (EXOS-D) satellite, J. Geomag. Geoelectr., 42, 555-564, 1990.

Østgaard, N., S. B. Mende, H. U. Frey, T. J. Immel, L. A. Frank, J. B. Sigwarth, and T. J. Stubbs, Interplanetary magnetic field control of the location of substorm onset and auroral features in the conjugate hemispheres, J. Geophys. Res., 109, A07204, doi:10.1029/2003JA010370, 2004.

Otsuka, Y., K. Shiokawa, T. Ogawa, and P. Wilkinson, Geomagnetic conjugate observations of medium-scale traveling ionospheric disturbances at midlatitude using all-sky airglow imagers, Geophys. Res. Lett., 31, L15803, doi:10.1029/2004GL020262, 2004.

Sagawa, E., T. Maruyama, T. J. Immel, H. U. Frey, and S. B. Mende, Global view of the nighttime low-latitude ionosphere by the IMAGE/FUV $135.6 \mathrm{~nm}$ observations, Geophys. Res. Lett., 30, 1534, doi:10.1029/2003GL017140, 2003.

Sasaki, S., Y. Iijima, K. Tanaka, M. Kato, M. Hashimoto, H. Mizutani, and Y. Takizawa, The SELENE mission: Goals and status, Adv. Space Res., 31, 2335-2340, 2003

Sato, N. and T. Saemundsson, Conjugacy of electron auroras, Mem. Natl. Inst. Polar Res., Special Issue, 48, 58-71, 1987.

Shiokawa, K., Y. Katoh, M. Satoh, M. K. Ejiri, T. Ogawa, T. Nakamura, T. Tsuda, and R. H. Wiens, Development of optical mesosphere thermosphere imagers (OMTI), Earth Planets Space, 51, 887-896, 1999.

Stenbaek-Nielsen, H. C., T. N. Davis, and N. W. Glass, Relative motion of auroral conjugate points during substroms, J. Geophys. Res., 77, 1844$1858,1972$.

Taylor, M. J. and M. A. Hapgood, On the origin of ripple-type structure in the OH nightglow emission, Planet. Space Sci., 38, 1421-1430, 1990.

Torr, M. R., D. G. Torr, M. Zukic, R. B. Johnson, J. Ajello, P. Banks, K Clark, K. Cole, C. Keffer, G. Parks, B. Tsurutani, and J. Spann, A far ultraviolet imager for the international solar-terrestrial physics mission, Space Sci. Rev., 71, 329-383, 1995.

Yoshikawa, I., A. Yamazaki, G. Murakami, K. Yoshioka, S. Kameda, F. Ezawa, T. Toyota, W. Miyake, M. Taguchi, M. Kikuchi, and M. Nakamura, Telescope of extreme ultraviolet (TEX) onboard SELENE: science from the Moon, Earth Planets Space, 60, 407-416, 2008.

M. Taguchi (e-mail: taguchi@rikkyo.ac.jp), T. Sakanoi, S. Okano, M. Kagitani, M. Kikuchi, M. Ejiri, I. Yoshikawa, A. Yamazaki, G. Murakami, K. Yoshioka, S. Kameda, W. Miyake, M. Nakamura, and K. Shiokawa 